\title{
Pressure Effects on the Transition Temperature and the Magnetic Field Penetration Depth in the Pyrochlore Superconductor $\mathrm{RbOs}_{2} \mathrm{O}_{6}$
}

\author{
R. Khasanov, ${ }^{1,2,3}$ D. G. Eshchenko, ${ }^{3,4}$ J. Karpinski, ${ }^{5}$ S. M. Kazakov, ${ }^{5}$ N. D. Zhigadlo, ${ }^{5}$ R. Brütsch, ${ }^{6}$ D. Gavillet, ${ }^{6}$ \\ D. Di Castro, ${ }^{3}$ A. Shengelaya, ${ }^{3}$ F. La Mattina, ${ }^{3}$ A. Maisuradze, ${ }^{3}$ C. Baines, ${ }^{4}$ and H. Keller ${ }^{3}$ \\ ${ }^{1}$ Laboratory for Neutron Scattering, Eidgenössische Technische Hochschule, Zürich, Switzerland, \\ and Paul Scherrer Institut, CH-5232 Villigen PSI, Switzerland \\ ${ }^{2}$ DPMC, Université de Genève, 24 Quai Ernest-Ansermet, 1211 Genève 4, Switzerland \\ ${ }^{3}$ Physik-Institut der Universität Zürich, Winterthurerstrasse 190, CH-8057, Zürich, Switzerland \\ ${ }^{4}$ Laboratory for Muon Spin Spectroscopy, Paul Scherrer Institute, CH-5232 Villigen PSI, Switzerland \\ ${ }^{5}$ Solid State Physics Laboratory, Eidgenössische Technische Hochschule, 8093 Zürich, Switzerland \\ ${ }^{6}$ Laboratory for Material Behaviour, Paul Scherrer Institut, CH-5232 Villigen PSI, Switzerland
}

(Received 22 April 2004; published 5 October 2004)

Magnetization measurements under hydrostatic pressure up to $8 \mathrm{kbar}$ in the pyrochlore superconductor $\mathrm{RbOs}_{2} \mathrm{O}_{6}\left(T_{c} \simeq 6.3 \mathrm{~K}\right.$ at $\left.p=0\right)$ were carried out. A positive pressure effect on $T_{c}$ with $d T_{c} / d p=0.090(3) \mathrm{K} / \mathrm{kbar}$ was observed, whereas no pressure effect on the magnetic penetration depth $\lambda$ was detected. The pressure independent ratio $2 \Delta_{0} / k_{B} T_{c}=3.72(2)\left(\Delta_{0}\right.$ is the superconducting gap at zero temperature) was found to be close to the BCS value 3.52. Magnetization and muon-spin rotation measurements of $\lambda(T)$ indicate that $\mathrm{RbOs}_{2} \mathrm{O}_{6}$ is an adiabatic $s$-wave BCS-type superconductor. The value of $\lambda$ extrapolated to zero temperature and ambient pressure was estimated to be 230(30) $\mathrm{nm}$.

DOI: 10.1103/PhysRevLett.93.157004

There is increasing interest in the physics of geometrically frustrated systems, especially after the observation of bulk superconductivity in the pyrochlore oxides $\mathrm{CdRe}_{2} \mathrm{O}_{7}\left(T_{c} \simeq 1 \mathrm{~K}\right)[1,2], \mathrm{KOs}_{2} \mathrm{O}_{6}\left(T_{c} \simeq 9.6 \mathrm{~K}\right)$ [3], $\mathrm{RbOs}_{2} \mathrm{O}_{6}\left(T_{c} \simeq 6.3 \mathrm{~K}\right)$ [4-7], and $\mathrm{CsOs}_{2} \mathrm{O}_{6}\left(T_{c} \simeq 3.3 \mathrm{~K}\right)$ [8]. The nature of the pairing mechanism in these pyrochlore compounds is still an open question. $\mathrm{CdRe}_{2} \mathrm{O}_{7}$ is suggested to be a weak-coupling BCS superconductor [9] without nodes in the superconducting gap $[9,10]$. Specific heat measurements performed on $\mathrm{RbOs}_{2} \mathrm{O}_{6}[5]$ are consistent with BCS-type behavior. On the other hand, Hiroi et al. [4] and Koda et al. [11] pointed to an unconventional mechanism of superconductivity in $\mathrm{KOs}_{2} \mathrm{O}_{6}$ and suggested that the superconducting order parameter is anisotropic [11].

Studies of the magnetic field penetration depth and experiments under pressure traditionally play an important role in superconductivity. The temperature dependence of the magnetic penetration depth $\lambda$ provides information on the nature of superconductivity, whereas the low-temperature behavior of $\lambda(T)$ reflects the superconducting gap structure. Additional information can be obtained from pressure studies on $T_{c}$ and other physical quantities.

In this letter we report magnetization studies of the hydrostatic pressure effect on the superconducting temperature $T_{c}(p)$ and the magnetic field penetration depth $\lambda(T, p)$ in the pyrochlore superconductor $\mathrm{RbOs}_{2} \mathrm{O}_{6}$. The temperature dependence of $\lambda$ at ambient pressure was also studied by means of muon-spin rotation ( $\mu \mathrm{SR})$ down to $T=30 \mathrm{mK}$.

Details of the sample preparation for $\mathrm{RbOs}_{2} \mathrm{O}_{6}$ can be found elsewhere $[5,6]$. Following the classical work of
PACS numbers: 74.70.Dd, 74.25.Ha, 74.62.Fj, 83.80.Fg

Shoenberg [12], for fine powders with known grain sizes the magnetic penetration depth can be calculated from the magnetic susceptibility $\chi$. The $\mathrm{RbOs}_{2} \mathrm{O}_{6}$ powder sample was ground and the grain-size distribution was determined by analyzing scanning electron microscope photographs. The distribution of the volume fraction $\sim N(R) R^{3}$ $[N(R)$ is the measured particle radius distribution] is shown in the inset of Fig. 1. The hydrostatic pressure

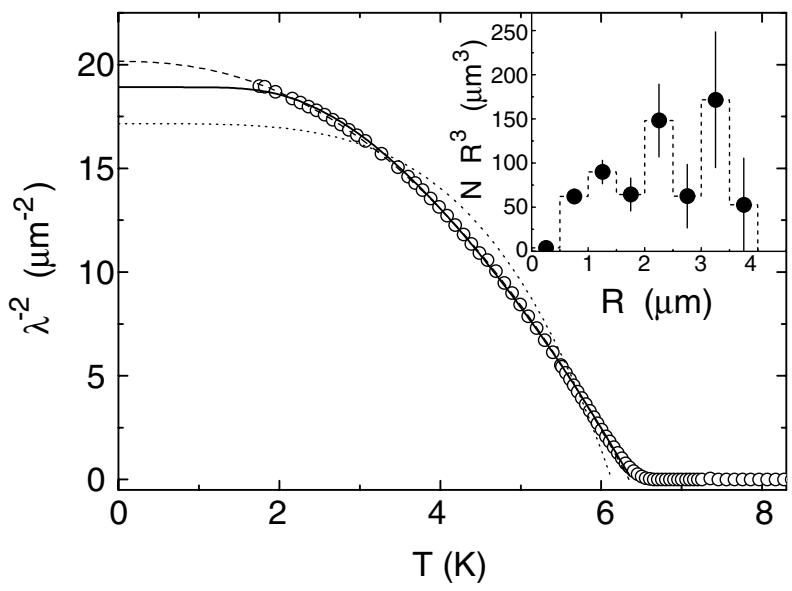

FIG. 1. Temperature dependence of $\lambda^{-2}$ for $\mathrm{RbOs}_{2} \mathrm{O}_{6}$ calculated from the measured $f(T)$ by using Eq. (1). Lines represent fit with the weak-coupling BCS model (solid line), strongcoupling BCS model (dotted line), and with a power law (dashed line). See text for an explanation. The inset shows the volume fraction distribution $N(R) R^{3}$ of the $\mathrm{RbOs}_{2} \mathrm{O}_{6}$ powder determined from scanning electron microscope photographs. The errors are statistical. 
was generated in a copper-beryllium piston cylinder clamp especially designed for magnetization measurements under pressure [13]. The sample was mixed with Fluorient FC77 (pressure transmitting medium) with a sample to liquid volume ratio of approximately $1 / 6$. The pressure was taken from a separate calibration set of magnetization experiments where a small piece of indium $\left[T_{c}(0)=3.4 \mathrm{~K}\right]$ with known $T_{c}(p)$ dependence was added to the sample and both $T_{c}$ 's of indium and $\mathrm{RbOs}_{2} \mathrm{O}_{6}$ were recorded.

The field-cooled (FC) and zero-field-cooled (ZFC) magnetization measurements were performed with a SQUID magnetometer in a field of $0.5 \mathrm{mT}$ at temperatures between $1.75 \mathrm{~K}$ and $10 \mathrm{~K}$. The absence of weak links between grains was confirmed by the linear magnetic field dependence of the FC magnetization, measured at $0.25,0.5$, and $1.0 \mathrm{mT}$ for each pressure at $T=1.75 \mathrm{~K}$. The temperature dependence of $\lambda$ was calculated from the measured ZFC magnetization by using the Shoenberg formula [12], modified for the known grain-size distribution $N(R)$ [14]:

$$
\begin{aligned}
\chi(T)= & -\frac{3}{2} \int_{0}^{\infty}\left(1-\frac{3 \lambda(T)}{R} \operatorname{coth} \frac{R}{\lambda(T)}\right. \\
& \left.+\frac{3 \lambda^{2}(T)}{R^{2}}\right) g(R) d R / \int_{0}^{\infty} g(R) d R,
\end{aligned}
$$

where $R$ is the grain radius, $g(R)=N(R) R^{3}$ (see inset in Fig. 1). The resulting temperature dependence $\lambda^{-2}(T, 0)$ at ambient pressure is shown in Fig. 1.

The reconstructed data were fitted with different models. The dotted line represents the fit with the two-fluid model $\quad \lambda^{-2}(T) / \lambda^{-2}(0)=1-\left(T / T_{c}\right)^{4} \quad\left[T_{c}=6.13(2) \mathrm{K}\right.$ and $\lambda(0)=241(5) \mathrm{nm}$ ] which corresponds to a strongcoupled BCS superconductor [15]. Here the errors are transferred from the "noise" of the magnetization measurements and do not include systematic errors which are discussed later. The solid line represents the best fit with the weak-coupling BCS model [15]:

$$
\frac{\lambda^{-2}\left(T, \Delta_{0}\right)}{\lambda^{-2}(0)}=1+2 \int_{\Delta(T)}^{\infty} \frac{\partial F}{\partial E} \frac{E}{\sqrt{E^{2}-\Delta(T)^{2}}} d E,
$$

where, $F=\left[1+\exp \left(E / k_{B} T\right)\right]^{-1}$ is the Fermi function, $\Delta(T)=\Delta_{0} \tilde{\Delta}\left(T / T_{c}\right)$ represents the temperature dependence of the energy gap, $\Delta_{0}$ is the zero temperature value of the superconducting gap, and $\tilde{\Delta}\left(T / T_{c}\right)$ is the normalized gap taken from Ref. [16]. A best fit to the data using Eq. (2) yields $T_{c}=6.35(2) \mathrm{K}, \lambda(0)=234(1) \mathrm{nm}$, and $\Delta_{0}=1.02(2) \mathrm{meV}$. At ambient pressure the ratio $2 \Delta_{0} / k_{B} T_{c}=3.73(6)$ is found to be close to the standard weak-coupling BCS value 3.52 [15]. For comparison with literature, we also analyzed the data with the empirical power law $\lambda^{-2}(T) / \lambda^{-2}(0)=1-\left(T / T_{c}\right)^{n}$ [17] (dashed line in Fig. 1), yielding $T_{c}=6.35(1), \lambda(0)=223(1) \mathrm{nm}$, and $n=2.20(1)$. A value of $n \approx 2$ was reported for
$\mathrm{KOs}_{2} \mathrm{O}_{6}$ which was interpreted as an indication for $d$-wave type of pairing [11].

To distinguish between $s$-wave and $d$-wave models one has to know $\lambda^{-2}(T)$ at low temperatures where they exhibit a completely different behavior (see Fig. 1). For this purpose we performed magnetic field penetration depth measurements down to $30 \mathrm{mK}$ using transversefield $\mu \mathrm{SR}$ (see e.g. [17]). The $\mu \mathrm{SR}$ experiments were performed at the Paul Scherrer Institute (PSI, Switzerland) on the $\pi \mathrm{M} 3$ beam line. In a powder sample the magnetic penetration depth $\lambda$ can be extracted from the muon-spin depolarization rate $\sigma(T) \propto 1 / \lambda^{2}(T)$ (see, e.g., [17]). For the measurements of $\sigma(T)$ the sample was field cooled from far above $T_{c}$ in a magnetic field of $1 \mathrm{~T}$. Figure 2 shows the normalized superfluid density $\lambda^{-2}(T) / \lambda^{-2}(0)=\sigma(T) / \sigma(0)$ versus reduced temperature $T / T_{c}$ for $0.5 \mathrm{mT}$ (low-field magnetization data) and $1 \mathrm{~T}$ ( $\mu$ SR data). In spite of the different nature (surface, Meissner state vs. bulk, mixed state) of the magnetization and $\mu$ SR measurements, the normalized $\lambda^{-2}(T)$ and $\sigma(T)$ data almost coincide over the whole temperature range where both types of measurements are available. The inset shows the low-temperature part of $\sigma(T)$ between $0 \mathrm{~K}$ and $1.3 \mathrm{~K}$. In this temperature range the depolarization rate $\sigma \propto 1 / \lambda^{2}$ is constant within the errors, suggesting that $\mathrm{RbOs}_{2} \mathrm{O}_{6}$ is a weak-coupled BCS $s$-wave superconductor. The solid line in Fig. 2 represents the fit of the $\mu \mathrm{SR}$ data by Eq. (2) with fixed $T_{c}=5.59 \mathrm{~K}$ obtained from the corresponding ( $1 \mathrm{~T}$ ) field-cooled magnetization measurements. The fit yields $\sigma(0)=$ 1.057(4) $\mu \mathrm{s}^{-1}$, and $\Delta_{0}=0.81(2) \mathrm{meV}$. The ratio $2 \Delta_{0} / k_{B} T_{c}=3.36(8)$ is close to the value 3.73(6) extracted from the magnetization measurements at ambient pressure. From $\sigma(0)$ measured by $\mu \mathrm{SR}$ and $H_{c_{2}}$ measured by

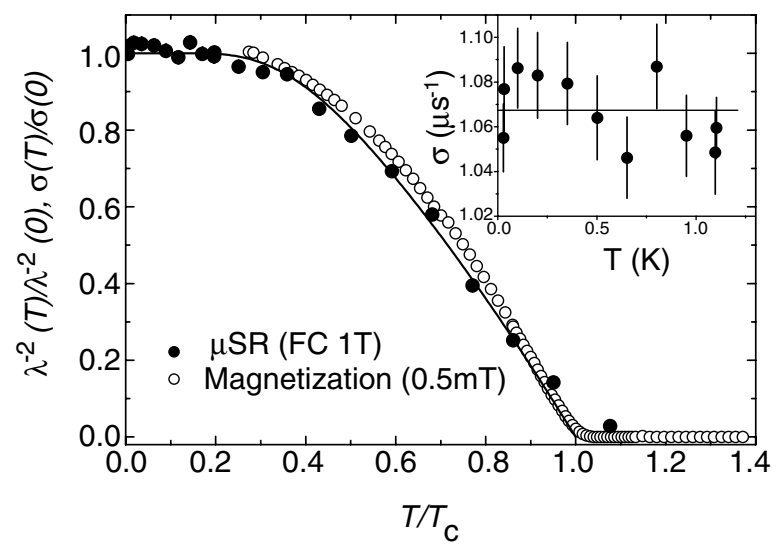

FIG. 2. Normalized superfluid density $\lambda^{-2}(T) / \lambda^{-2}(0)=$ $\sigma(T) / \sigma(0)$ versus reduced temperature $T / T_{c}$ for $0.5 \mathrm{mT}$ (lowfield magnetization) and $1 \mathrm{~T}(\mu \mathrm{SR})$. The solid line represents the fit to the $\mu \mathrm{SR}$ data with Eq. (2). The inset shows the lowtemperature region between $0 \mathrm{~K}$ and $1.3 \mathrm{~K}$; the dashed line represents the average value. 
magnetization, the value of $\lambda(0)$ at ambient pressure is estimated to be 240(30) $\mathrm{nm}$. Details of this procedure will be published elsewhere [18].

A rough estimate of the uncertainty in the absolute value of $\lambda(0)$ reconstructed from magnetization measurements and introduced by the statistical nature of the grain-size distribution was done by calculating $\lambda(T)$ for $N(R)+\sqrt{N(R)}$ and $N(R)-\sqrt{N(R)}$ distributions. The fit of the resulting $\lambda(T)$ curves with the weak-coupling BCS model gives $\lambda(0)$ in the range from 200 to $355 \mathrm{~nm}$. An additional source of uncertainty comes from the deviation of the grains' shape from a spherical one. In the assumption of the small deviation of the demagnetization factor of the real grain from the demagnetization factor of the spherical grain $(1 / 3 \pm 10 \%)$, the relative error of $\lambda(0)$ is of the order of $3 \%$. Finally, the interval for the $\lambda(0)$ can be estimated ranging from 195 to $260 \mathrm{~nm}$, in accordance with $\lambda(0)=240$ (30) nm extracted from more direct $\mu \mathrm{SR}$ measurements.

In the following the pressure effect measurements on $T_{c}$ and $\lambda$ are discussed. In these experiments, we studied relative effects measured on the same sample in the same pressure cell. The main systematic error of these measurements comes from misalignments of the experimental setup after the cell was removed from the SQUID magnetometer and put back again. This procedure was checked with a set of measurements at constant pressure. The systematic scattering of the magnetization data is about $0.5 \%$, giving a relative error in $\lambda^{-2}(T)$ of about $2 \%$. The pressure data of $\lambda(T)$ reveal that the transition tem-

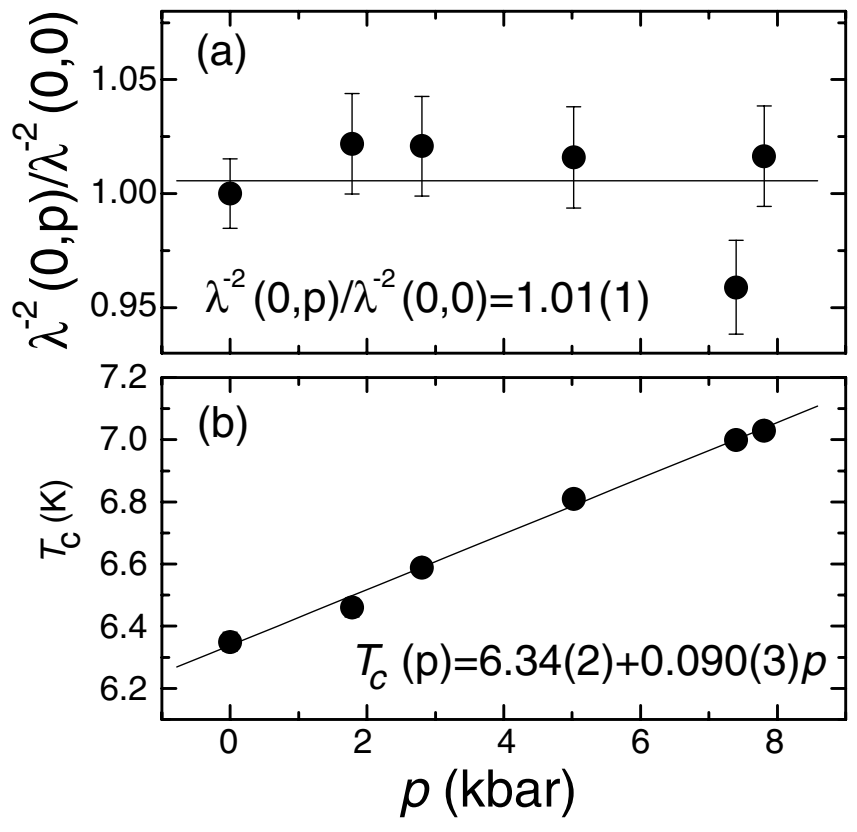

FIG. 3. Pressure dependence of (a) $\lambda^{-2}(0)(p) / \lambda^{-2}(0)(p=0)$ and (b) $T_{c}$. The solid lines are fits with parameters shown in the figure. perature increases almost by $1 \mathrm{~K}$ at $8 \mathrm{kbar}$, whereas the reconstructed $\lambda(T, p)$ curves are indistinguishable within the error bars after $T / T_{c}$ scaling. This implies that $\lambda(0)$ is pressure independent, suggesting that $\mathrm{RbOs}_{2} \mathrm{O}_{6}$ is an adiabatic superconductor, in contrast to cuprate superconductors [19]. The values of $\lambda(0), T_{c}$, and $\Delta_{0}$ were extracted from the fit of the reconstructed $\lambda(T)$ data with the weak-coupling BCS model [Eq. (2)]. The $\lambda^{-2}(0, p) / \lambda^{-2}(0,0)$ data are scattered and touching by error bars [see Fig. 3(a)]. Note that recently no substantial pressure effect on $\lambda(0)$ was also observed in $\mathrm{MgB}_{2}$ [20] which is accepted to be a phonon mediated superconductor.

The pressure dependence of the critical temperature $T_{c}$ is shown in Fig. 3(b). The linear fit yields $d T_{c} / d p=$ $0.090(3) \mathrm{K} / \mathrm{kbar}$. The results on the zero temperature superconducting gap $\Delta_{0}$ are summarized in Fig. 4, for $\Delta_{0}$ is plotted as a function of the critical temperature $T_{c}$. The solid line represents the best fit by the relation $\Delta_{0} \propto$ $T_{c}$ to the magnetization data. The proportional relation between $\Delta_{0}$ and $T_{c}$ can be understood in the frame of the BCS theory which predicts the universal ratio $2 \Delta_{0} / k_{B} T_{c}=3.52$. In the present studies this ratio was found to be pressure independent within experimental errors with the mean value 3.72(2).

For the majority of BCS-type superconductors (including $\left.\mathrm{MgB}_{2}\right) T_{c}$ decreases with increasing pressure. This is in contrast to the observed positive pressure effect on $T_{c}$ in the $\mathrm{RbOs}_{2} \mathrm{O}_{6}$ [Fig. 3(b)]. The pressure shift on $T_{c}$ is derived to be [21]:

$$
\frac{d \ln T_{c}}{d p}=-(2 A-1) \frac{d \ln \langle\omega\rangle}{d p}+A \frac{d \ln \eta}{d p},
$$

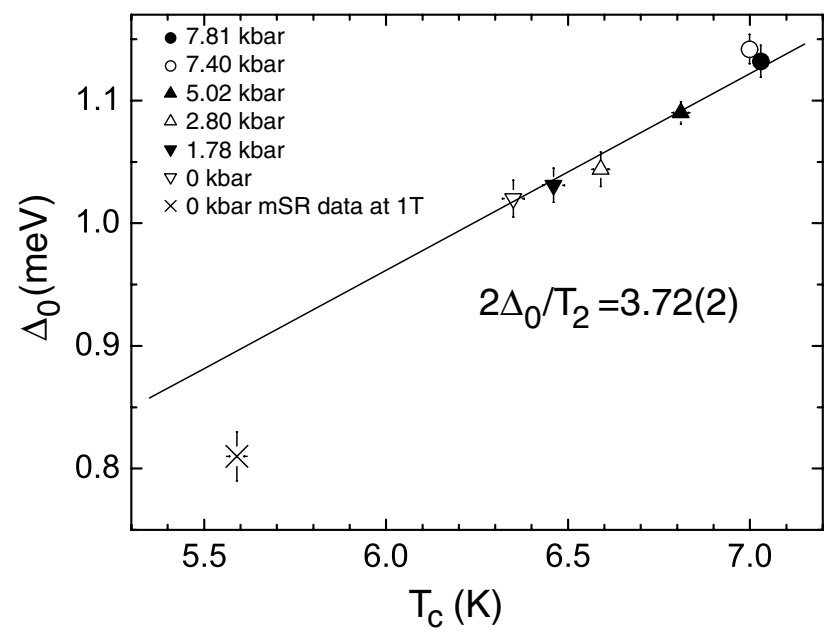

FIG. 4. $\Delta_{0}$ vs. $T_{c}$ in $\mathrm{RbOs}_{2} \mathrm{O}_{6}$. Both magnetization data under pressure (circles and triangles) and $\mu \mathrm{SR}$ data (cross) are shown. Note that $T_{c}$ for the $\mu \mathrm{SR}$ point is lower due to the high magnetic field $1 \mathrm{~T}$. The solid line represents the best fit with the relation $\Delta_{0} \propto T_{c}$. 
where $A$ is a function of the electron-phonon coupling constant $\lambda_{e l-p h}$ and the Coulomb pseudopotential $\mu^{*}$ [21], $\langle\omega\rangle$ is an average phonon frequency, $\eta \equiv N\left(E_{f}\right) \times$ $\left\langle I^{2}\right\rangle$ is the Hopfeld parameter [22], $N\left(E_{f}\right)$ is the density of states at the Fermi level, and $\left\langle I^{2}\right\rangle$ is the average squared electronic matrix element. There are two contributions to the pressure shift on $T_{c}$ : (1) a phononic contribution $(d \ln \langle\omega\rangle / d p)$ and (2) an electronic contribution $\left(d \ln N\left(E_{f}\right)\left\langle I^{2}\right\rangle / d p\right)$. The effect of pressure on the phonon spectrum usually results in an increase of the average phonon frequency. In rare cases close to a structural transition phonon "softening" can occur, but usually only for some particular modes [23]. For conventional superconductors (including $\mathrm{MgB}_{2}$ ) typical values of $A$ are in the range $0.5-8.0[21,24]$, so the first term of Eq. (3) is generally negative. Since we observed a positive pressure effect on $T_{c}$, the second term of Eq. (3) should be positive, which is generally observed $[22,25]$. This suggests that the observed pressure effect on $T_{c}$ arises from the electronic term. A similar effect was also observed in $\mathrm{Cd}_{2} \mathrm{Re}_{2} \mathrm{O}_{7}$ [26] with $d T_{c} / d p=0.062 \mathrm{~K} / \mathrm{kbar}$, comparable with the value reported here. A probable scenario of this effect may be a sort of charge ordering, resulting in the disproportionation of the Os tetrahedra as discussed for $\mathrm{CdRe}_{2} \mathrm{O}_{7}$ in [27]. However, for a more quantitative description of $T_{c}(p)$ additional experimental and theoretical work is needed.

In conclusion, we performed magnetization measurements in $\mathrm{RbOs}_{2} \mathrm{O}_{6}$ under hydrostatic pressure. A positive pressure effect on $T_{c}$ with $d T_{c} / d p=0.090(3) \mathrm{K} / \mathrm{kbar}$ is observed, in contrast to the negative pressure shift generally detected in conventional superconductors. The absence (within the experimental uncertainties) of a pressure effect on $\lambda$ suggests that $\mathrm{RbOs}_{2} \mathrm{O}_{6}$ is an adiabatic BCS-type superconductor. The ratio $2 \Delta_{0} / k_{B} T_{c}=3.72(2)$ is found to be pressure independent and close to the BCS value 3.52. The magnetization and $\mu \mathrm{SR}$ measurements both indicate that the temperature dependence of $\lambda$ is consistent with that expected for a weak-coupled $s$-wave BCS superconductor. The value of $\lambda$ extrapolated to zero temperature and ambient pressure was estimated to be 230(30) $\mathrm{nm}$.

This work was partly performed at the Swiss Muon Source (S $\mu \mathrm{S})$, Paul Scherrer Institute (PSI, Switzerland). The authors are grateful to A. Amato and D. Herlach for providing beam time within the PSI short-term proposal system, and C. Grimaldi for helpful discussions. This work was supported by the Swiss National Science Foundation and by the NCCR program Materials with Novel Electronic Properties (MaNEP) sponsored by the Swiss National Science Foundation.
[1] M. Hanawa, Y. Muraoka, T. Tayama, T. Sakakibara, J. Yamaura, and Z. Hiroi, Phys. Rev. Lett. 87, 187001 (2001).

[2] H. Sakai, K. Yoshimura, H. Ohno, H. Kato, S. Kambe, R. E. Walstedt, T. D. Matsuda, Y. Haga, and Y. Onuki, J. Phys. Condens. Matter 13, L785 (2001).

[3] S. Yonezawa, Y. Muraoka, Y. Matsushita, and Z. Hiroi, J. Phys. Condens. Matter 16, L9 (2004).

[4] Z. Hiroi, S. Yonezawa, and Y. Muraoka, J. Phys. Soc. Jpn. 73, 1651 (2004).

[5] M. Brühwiler, S. M. Kazakov, N. D. Zhigadlo, J. Karpinski, and B. Batlogg, Phys. Rev. B 70, 020503(R) (2004).

[6] S. M. Kazakov, N. D. Zhigadlo, M. Brühwiler, B. Batlogg, and J. Karpinski, Supercond. Sci. Technol. 17, 1169 (2004).

[7] S. Yonezawa, Y. Muraoka, Y. Matsushita, and Z. Hiroi, J. Phys. Soc. Jpn. 73, 819 (2004).

[8] S. Yonezawa, Y. Muraoka, and Z. Hiroi, cond-mat/ 0404220 .

[9] Z. Hiroi and M. Hanawa, J. Phys. Chem. Solids 63, 1021 (2002).

[10] M. D. Lumsden, S. R. Dunsiger, J. E. Sonier, R. I. Miller, R. F. Kiefl, R. Jin, J. He, D. Mandrus, S. T. Bramwell, and J. S. Gardner, Phys. Rev. Lett. 89, 147002 (2001).

[11] A. Koda, W. Higemoto, K. Ohishi, S. R. Saha, R. Kadono, S. Yonezawa, Y. Muraoka, and Z. Hiroi, cond-mat/ 0402400.

[12] D. Shoenberg, Proc. R. Soc. London A 175, 49 (1940).

[13] T. Straessle, Ph.D. thesis, ETH Zurich, 2001.

[14] A. Porch, J. R. Cooper, D. N. Zheng, J. R. Waldram, A. M. Campbell, and P. A. Freeman, Physica C (Amsterdam) 214, 350 (1993).

[15] M. Tinkham, Introduction to Superconductivity (Krieger Publishing Company, Malabar, Florida, 1975).

[16] B. Mühlschlegel, Z. Phys. 155, 313 (1959).

[17] P. Zimmermann, H. Keller, S. L. Lee, I. M. Savic, M. Warden, D. Zech, R. Cubitt, E. M. Forgan, E. Kaldis, J. Karpinski, and C. Krüger, Phys. Rev. B 52, 541 (1995).

[18] R. Khasanov et al. (to be published).

[19] R. Khasanov, J. Karpinski, and H. Keller, cond-mat/ 0405643.

[20] D. Di Castro et al., (unpublished).

[21] T. Tomita, J. J. Hamlin, J. S. Schilling, D. G. Hinks, and J. D. Jorgensen, Phys. Rev. B 64, 092505 (2001).

[22] J. J. Hopfeld, Physica (Amsterdam) 55, 41 (1971).

[23] A. L. Chen, S. P. Lewis, Z. Su, P. Y. Yu, and M. L. Cohen, Phys. Rev. B 46, 5523 (1992).

[24] J. P. Carbotte, Rev. Mod. Phys. 62, 1027 (1990).

[25] J. S. Schilling and S. Klotz, Physical Properties of High Temperature Superconductors, (World Scientific, Singapore, 1992), Vol. III, p. 59.

[26] M. Abliz, M. Hedo, Z. Hiroi, T. Matsumoto, M. Hanawa, and Y. Uwatoko, J. Phys. Soc. Jpn. 72, 3039 (2003).

[27] Z. Hiroi, T. Yamauchi, T. Yamada, M. Hanawa, Y. Ohishi, O. Shimomura, M. Abliz, M. Hedo, and Y. Uwatoko, J. Phys. Soc. Jpn. 71, 1553 (2002). 